\title{
High-Performance Transparent Conducting Oxide Nanowires
}

\author{
Qing Wan, ${ }^{\dagger}$ Eric N. Dattoli, ${ }^{\dagger}$ Wayne Y. Fung, ${ }^{\dagger}$ Wei Guo, ${ }^{\ddagger}$ Yanbin Chen, ${ }^{\ddagger}$ \\ Xiaoqing Pan, ${ }^{\ddagger}$ and Wei $\mathrm{Lu}^{*, \dagger}$ \\ Department of Electrical Engineering and Computer Science and \\ Department of Materials Science and Engineering, University of Michigan, \\ Ann Arbor, Michigan 48109
}

Received September 20, 2006

\begin{abstract}
We report the growth and characterization of single-crystalline Sn-doped $\ln _{2} \mathrm{O}_{3}$ (ITO) and Mo-doped $\ln _{2} \mathrm{O}_{3}$ (IMO) nanowires. Epitaxial growth of vertically aligned ITO nanowire arrays was achieved on ITO/yttria-stabilized zirconia (YSZ) substrates. Optical transmittance and electrical transport measurements show that these nanowires are high-performance transparent metallic conductors with transmittance of $\sim 85 \%$ in the visible range, resistivities as low as $6.29 \times 10^{-5} \Omega \cdot \mathrm{cm}$ and failure-current densities as high as $3.1 \times 10^{7} \mathrm{~A} / \mathrm{cm}^{2}$. Such nanowires will be suitable in a wide range of applications including organic light-emitting devices, solar cells, and field emitters. In addition, we demonstrate the growth of branched nanowire structures in which semiconducting $\ln _{2} \mathrm{O}_{3}$ nanowire arrays with variable densities were grown epitaxially on metallic ITO nanowire backbones.
\end{abstract}

One-dimensional (1D) nanostructures such as nanowires, nanorods, and nanobelts have become the focus of intensive investigation in the past decade as potential building blocks for nanoscale devices and sensors. ${ }^{1-5}$ Along with group IV and III $-\mathrm{V}$ materials, metal oxide (including $\mathrm{In}_{2} \mathrm{O}_{3}, \mathrm{SnO}_{2}$ and $\mathrm{ZnO}$ ) nanowires have been widely studied due to their excellent electrical and optical properties and ease of fabrication. ${ }^{6-8}$ In these studies the metal oxide nanowires are typically not intentionally doped, and the carriers are normally generated by structural defects such as oxygen deficiencies. As a result, the devices behave as wide band gap semiconductors whose performance is influenced by the surrounding environment. ${ }^{8}$ On the other hand, intentional doping can greatly modify the device properties and yield new device applications. One such example is tin-doped indium oxide (ITO), in which metal-like behavior is achieved when $\mathrm{In}_{2} \mathrm{O}_{3}$ is degenerately doped by $\mathrm{Sn}$. Due to its high conductivity and high transmittance in the visible spectral region, ${ }^{9}$ ITO has become by far the most important transparent conducting oxide material, and ITO films have found applications in various optoelectronic devices such as flatpanel displays, solar cells, and light-emitting diodes. ${ }^{10-12}$ The ability to obtain highly transparent and highly conducting ITO nanowires may potentially further enhance the performance of such devices due to the increased effective device area using nanowire electrodes. Furthermore, similar to NiSi

\footnotetext{
* Corresponding author. E-mail: wluee@eecs.umich.edu.

Department of Electrical Engineering and Computer Science.

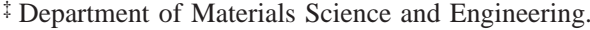

and $\mathrm{TaSi}_{2}$ nanowires, ${ }^{13,14}$ the highly conducting ITO nanowires may also be used as interconnects in integrated nanocsale devices.

The growth of ITO nanowires/nanorods has been reported by several groups since the first study on $\mathrm{In}_{2} \mathrm{O}_{3}$ nanobelts in 2001. ${ }^{15-21}$ However, detailed electrical characterizations have not been reported, and it is not clear whether these ITO nanowire/nanorods have the desired electrical properties. For example, the only reported resistivity value is $\sim 0.4 \Omega \cdot \mathrm{cm},{ }^{18}$ which is several orders higher than that can be obtained in commercially available ITO films ${ }^{9}$ and clearly too high for the nanowires to be used as electrodes. Here we report the growth and characterization of single crystalline Sn-doped $\mathrm{In}_{2} \mathrm{O}_{3}$ (ITO) and Mo-doped $\mathrm{In}_{2} \mathrm{O}_{3}$ (IMO) nanowires with very low resistivities and very high failure-current densities. Furthermore, we were able to obtain vertically aligned ITO and IMO nanowire arrays on lattice-matched ITO substrates. Compared to the randomly distributed nanowires reported in earlier studies, such vertical nanowire arrays are likely more suitable for applications such as solar cells due to the direct electrical pathway the ITO nanowires provide to the underlying ITO thin film electrode. Finally, we demonstrate that semiconducting $\mathrm{In}_{2} \mathrm{O}_{3}$ nanowires arrays can be epitaxially grown on highly conducting, metallic-like ITO nanowire backbones, offering a structure that may present a rational route for three-dimensional integration of nanowire devices.

The ITO nanowires were grown via a catalyst-mediated vapor-liquid-solid (VLS) method as described below. An 


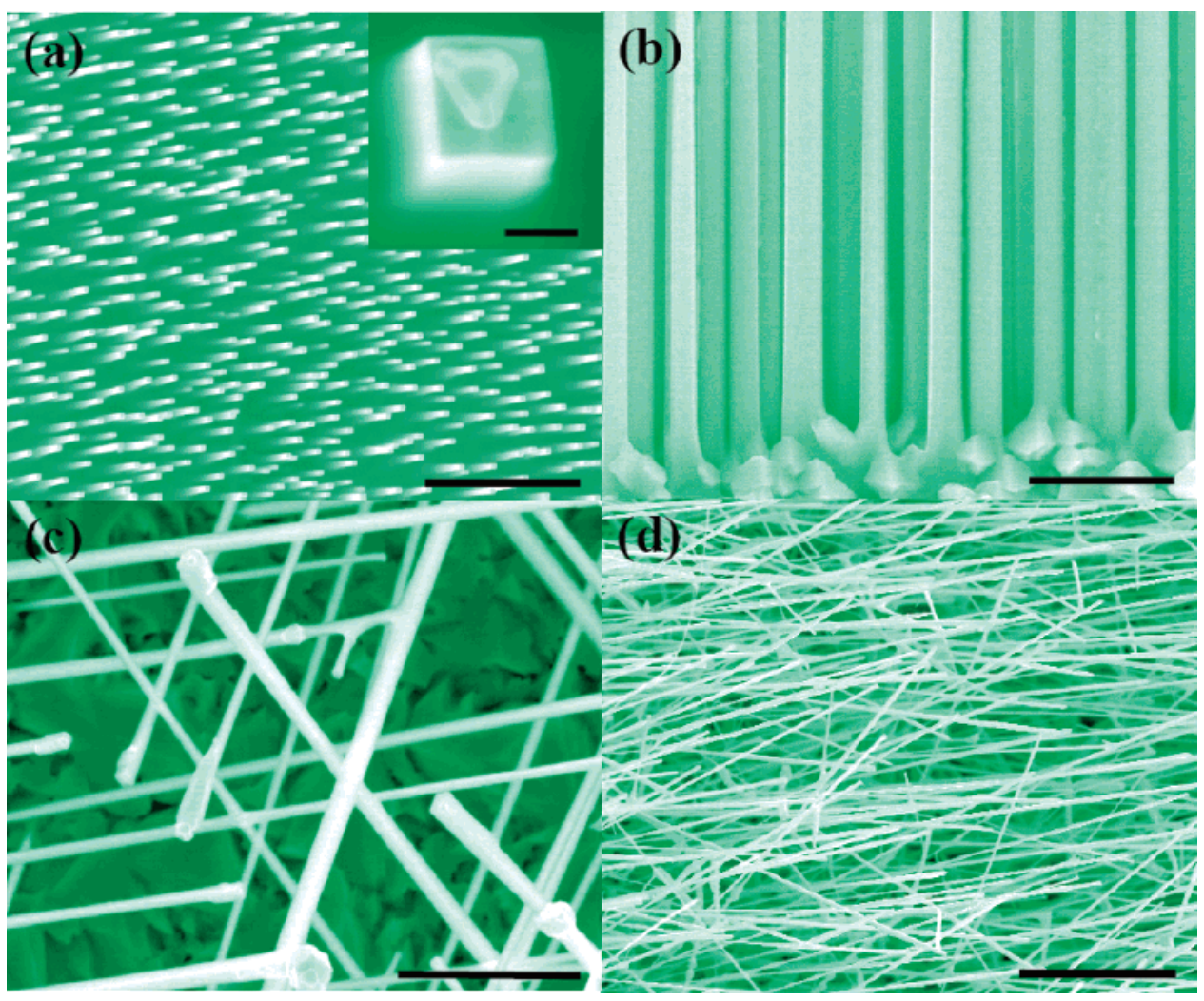

Figure 1. SEM images of as-synthesized ITO nanowires. (a) Top-view SEM image of an ITO nanowire array grown on an ITO/YSZ substrate. Scale bar: $5 \mu \mathrm{m}$. Inset: high-magnification top-view SEM image of a single ITO nanowire. Scale bar: $50 \mathrm{~nm}$. (b) Side-view SEM image of the ITO nanowire array showing the epitaxial, vertical growth of the nanowire array from the substrate. Scale bar: $500 \mathrm{~nm}$. (c) Top-view SEM image of ITO nanowires grown on a (111) YSZ substrate without the ITO buffer layer showing the weblike structure with 3-fold symmetry. Scale bar: $1 \mu \mathrm{m}$. (d) SEM image of ITO nanowires grown randomly on (100) Si substrate. Scale bar: $2 \mu \mathrm{m}$.

ITO (In:Sn $=95: 5)$ buffer layer was first deposited on a (100) YSZ $\left(1 \times 1 \mathrm{~cm}^{2}\right)$ substrate by a pulsed laser deposition (PLD) method. The single-crystalline ITO layer is lattice matched with the YSZ substrate and provides an ideal substrate for the subsequent ITO nanowire growth (see Supporting Information). A gold (Au) film with thickness of $10 \mathrm{~nm}$ was then deposited on the ITO/YSZ substrate by sputter deposition. At elevated temperatures the Au film coalesces into Au nanoclusters which in turn act as catalysts during the VLS nanowire growth scheme. Finally, high-purity $(99.99 \%)$ In and $\mathrm{SnO}$ powders were mixed thoroughly with atomic ratio of 90:10. The mixture and substrates were then loaded in an alumina boat and positioned at the center of an alumina tube that was inserted into a horizontal tube furnace. The growth temperature was $900{ }^{\circ} \mathrm{C}$ and the growth lasted $2 \mathrm{~h}$ under a $0.5 \mathrm{~L} / \mathrm{min}$ flux of nitrogen with a trace amount of oxygen.

Scanning electron microscopy (SEM) images of the assynthesized ITO nanowires clearly show the formation of vertically aligned nanowire arrays on the (100) ITO/(100) YSZ substrate, as depicted in parts a (top view) and b (side view) of Figure 1. Furthermore, the ITO nanowires exhibit a square cross section, as shown in the inset of Figure 1a, consistent with conditions for epitaxial growth along the (100) direction of single-crystalline ITO (bixbyite, cubic structure with lattice constant $a=1.01 \mathrm{~nm}$ ). The catalyst nanoparticle is also visible at the tip of the nanowire. The nanowires shown in parts a and $b$ of Figure 1 have a mean cross-sectional size of $\sim 100 \mathrm{~nm} \times 100 \mathrm{~nm}$, while nanowires with smaller or larger sizes can be achieved by adjusting the thickness of the Au catalyst films. We found that the ITO buffer layer improves the stability of the ITO nanowire nucleation and is critical for ensuring the growth of vertically aligned ITO nanowire arrays. For example, on bare (100) YSZ substrates without the ITO buffer layer, ITO nanowires with $\langle 111\rangle$ growth directions were observed at growth temperatures $>900{ }^{\circ} \mathrm{C}$, resulting in a weblike structure with 4-fold symmetry instead of vertical arrays. ${ }^{21}$ On the other hand, when (111) YSZ was used as the substrate, ITO nanowires with (100) growth directions were always observed due to the formation of YSZ $\{100\}$ facets, and resulted in a 3-fold geometric symmetry, as shown in Figure 1c. Finally, high-density albeit randomly distributed ITO nanowires were obtained if (100) $\mathrm{Si}$ was used as the substrate, as shown in Figure 1d.

Further structural characterizations were carried out on individual ITO nanowires by transmission electron microscopy (TEM). Figure 2a shows a typical energy dispersive $\mathrm{X}$-ray spectroscopy (EDS) spectrum of a single ITO nanowire. The presence of Sn was clearly observed along with $\mathrm{O}$ and In, and the atomic ratio of Sn:In was estimated to be $\sim 4: 100$ from the EDS spectrum. The other peaks in the EDS spectrum correspond to $\mathrm{C}$ and $\mathrm{Cu}$ that are present in the TEM grid used to support the nanowire. Figure $2 \mathrm{~b}$ shows a lowmagnification TEM image of an ITO nanowire with a lateral size of $100 \mathrm{~nm}$, along with the corresponding selected area 

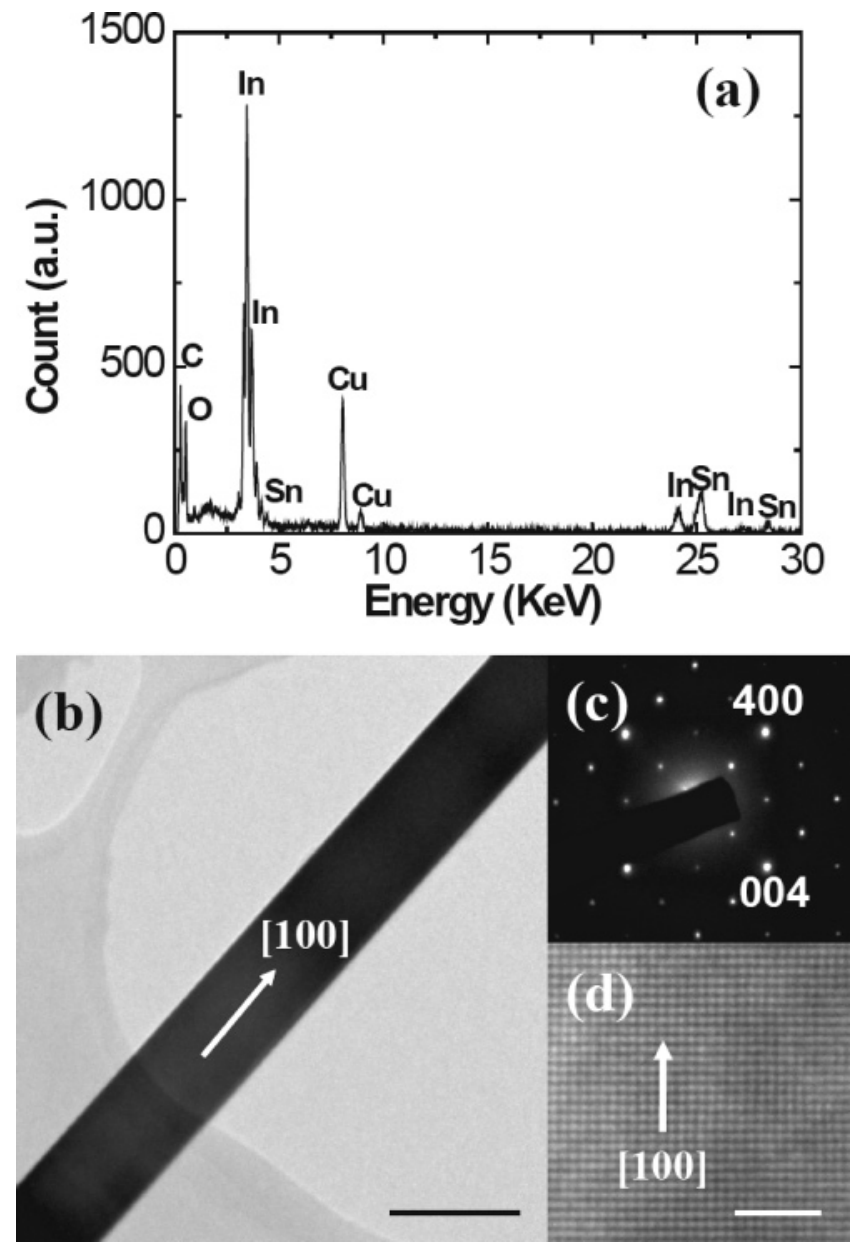

Figure 2. (a) Energy dispersive X-ray spectrum of a single, isolated ITO nanowire. a.u., arbitrary units. (b) Low-magnification TEM image of an ITO nanowire. Scale bar: $100 \mathrm{~nm}$. (c) Selected area diffraction pattern of the ITO nanowire shown in b. (d) HRTEM image of the same ITO nanowire. Scale bar: $4 \mathrm{~nm}$.

electron diffraction (SAED) pattern (Figure 2c). The indexed diffraction spots, 400 and 004, verify that the ITO nanowire has a cubic crystal structure and that the growth direction of the ITO nanowire is [100], consistent with the SEM observations. The lattice constant of the ITO nanowires was calculated to be $10.16 \AA$, also consistent with literature values. High-resolution TEM (HRTEM) characterizations of the ITO nanowires (Figure 2d) further verify the singlecrystalline nature of the nanowires and the absence of line or planar defects.

To probe the optical transmittance of the ITO nanowires, a nanowire film with thickness of $\sim 500 \mathrm{~nm}$ was formed on a glass substrate using a spin-coating method. The optical transmission spectrum of the ITO nanowire/glass system was recorded by a UV-visible spectrophotometer over the wavelength range of $200-800 \mathrm{~nm}$ at normal incidence. As shown in Figure 3, a transmittance of $65 \%$ in the visible light range $(380-800 \mathrm{~nm})$ was observed on the ITO nanowire/glass system (curve a). After subtraction of the influence of the glass substrate (curve b), a transmittance of about $85 \%$ (curve c) was obtained for the $500 \mathrm{~nm}$ thick ITO nanowire film. We note this high transmittance value is

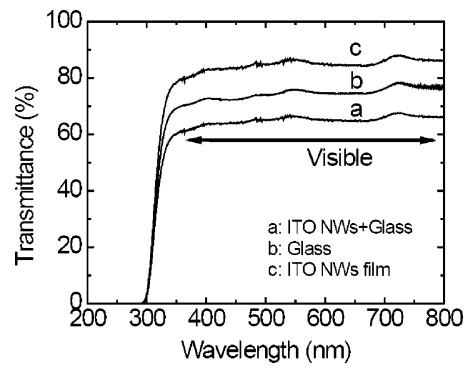

Figure 3. Optical transmittance spectrum of a $500 \mathrm{~nm}$ thick ITO nanowire film deposited on glass slides, showing the transmittances of the ITO/glass system (curve a), the glass slides alone (curve b), and the calculated transmittance of the ITO nanowire film (curve c).
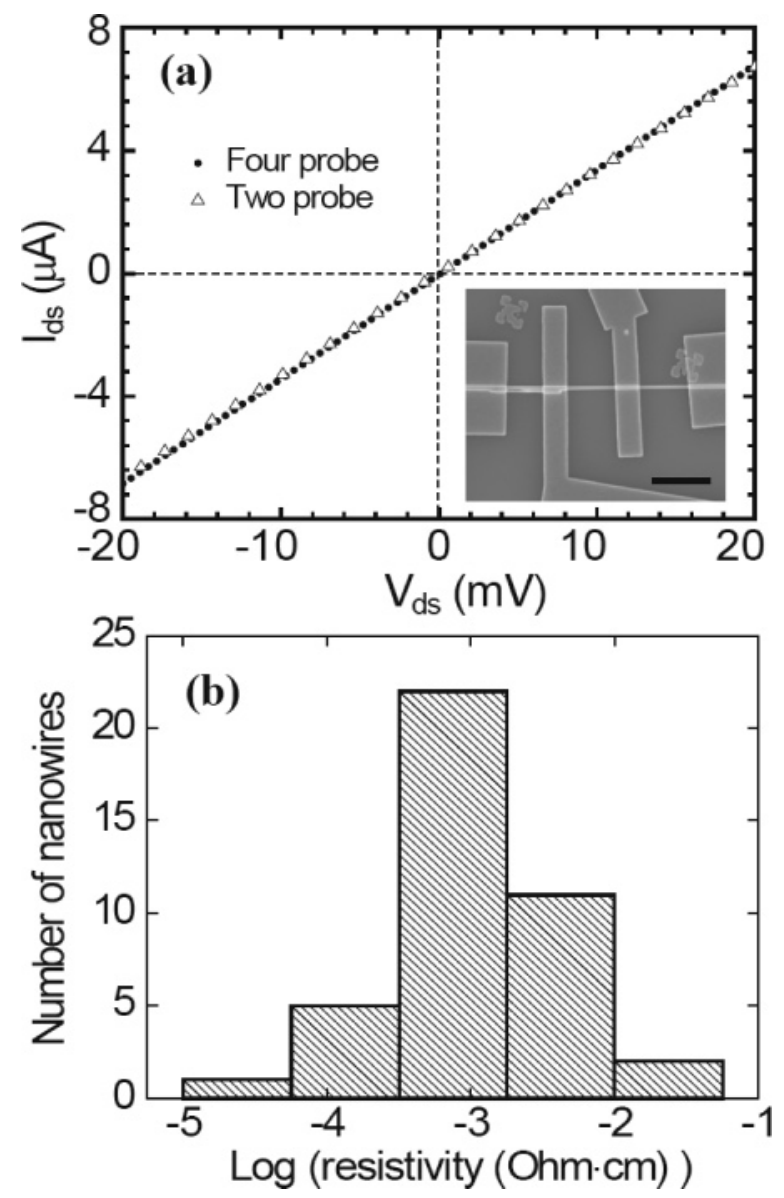

Figure 4. (a) Comparison of the current-voltage characteristics of an individual ITO nanowire device using four-probe and twoprobe configurations. Inset, SEM image of the ITO nanowire device. Scale bar: $2 \mu \mathrm{m}$. (b) Histogram of the resistivities measured over 40 ITO nanowire devices.

comparable with that reported on optimized transparent conducting oxides films of comparable thickness. ${ }^{22}$

The electrical transport properties of the ITO nanowires were studied on devices consisting of individual nanowires. The nanowires were first removed from the growth substrate by sonication in isopropyl alcohol and deposited onto a degenerately doped n-type silicon substrate capped with a $50 \mathrm{~nm}$ silicon dioxide layer. Photolithography or electron-beam (e-beam) lithography processes were used to define pairs of metal electrodes on the $\mathrm{SiO}_{2} / \mathrm{Si}$ substrate, 

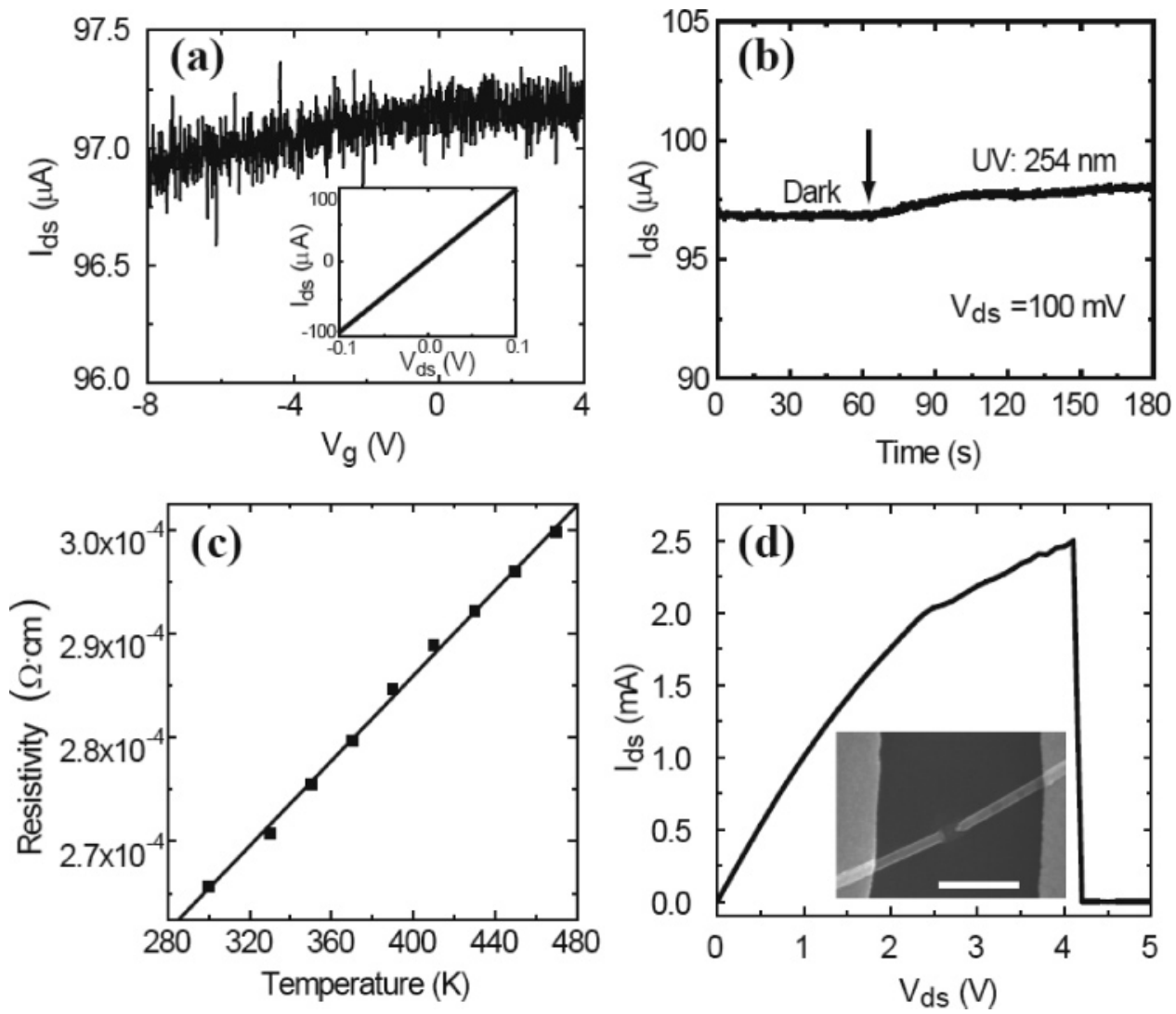

Figure 5. Electrical characterization of a single ITO nanowire device. (a) Transfer curve $\left(I_{\mathrm{ds}}-V_{\mathrm{g}}\right)$ of the ITO nanowire device at $V_{\mathrm{ds}}=100$ $\mathrm{mV}$. Inset, $I_{\mathrm{ds}}-V_{\mathrm{ds}}$ curve of the device. The two-probe resistance of the device is $\sim 1.05 \mathrm{k} \Omega$. (b) UV response of the same device. (c) Temperature-dependent resistivity values of the ITO nanowire $(300-470 \mathrm{~K})$. The solid line is a linear fit. (d) $I_{\mathrm{ds}}-V_{\mathrm{ds}}$ curve for the same ITO nanowire at large biases. The nanowire can carry a current of more than $2.5 \mathrm{~mA}$ before breaking down. Inset, SEM image of the failed ITO nanowire, showing that failure occurs in the middle of the nanowire. Scale bar: $500 \mathrm{~nm}$.

followed by metal deposition of Ti/Au $(10 \mathrm{~nm} / 100 \mathrm{~nm})$ by electron beam evaporation to complete the device structure. Prior to metal evaporation, the samples were cleaned with $\mathrm{O}_{2}$ plasma $(50 \mathrm{~W}, 30-60 \mathrm{~s})$ to remove possible resist residue. Postannealing processes were not performed in the devices.

More than 40 ITO nanowire devices were fabricated and the electrical properties of individual nanowires were investigated both in ambient air and in vacuum $\left(5 \times 10^{-5}\right.$ Torr $)$. We would like to emphasize that a linear current $\left(I_{\mathrm{ds}}\right)$ versus voltage $\left(V_{\mathrm{ds}}\right)$ curve (Figure 4a) was observed in all measurements and on samples defined by photolithography as well as e-beam lithography methods. This indicates that good Ohmic contacts can be readily achieved between the ITO nanowire and $\mathrm{Ti} / \mathrm{Au}$ electrodes. To further characterize this observation, four-probe measurements were carried out to study the effects of the contacts. As shown in Figure 4a, the $I_{\mathrm{ds}}-V_{\mathrm{ds}}$ curve obtained from the four-probe method is almost identical to that obtained from the two-probe method using the inner pair of electrodes. The small difference between the measured resistances $2936 \Omega$ (four-probe) and $2984 \Omega$ (two-probe) can be mostly attributed to the resistance of the metal leads $(\sim 55 \Omega)$ connecting the nanowire devices to the contact pads, and verifies that contacts to the ITO nanowires are indeed Ohmic with negligible values of resistance. The ability to produce reliable Ohmic contacts is very desirable in nanoscale device applications and also affords us to focus our studies on the simpler two-probe device structure in the following discussions.

From the measured resistance value of the device in Figure $4 \mathrm{a}$ and the cross-section size $(75 \mathrm{~nm})$ and the length $(1.8$ $\mu \mathrm{m}$ ) of the ITO nanowire (measured by SEM imaging), we can calculate the resistivity of the ITO nanowire to be 9.18 $\times 10^{-4} \Omega \cdot \mathrm{cm}$. The resistivities of about 40 ITO nanowires were obtained and plotted in Figure 4b. Significantly, the median resistivity value $7.15 \times 10^{-4} \Omega \cdot \mathrm{cm}$, and lowest resistivity value $6.29 \times 10^{-5} \Omega \cdot \mathrm{cm}$, are comparable to the best values achieved in high-quality ITO films ${ }^{9}$ and are several orders better than those reported for ITO nanowires in earlier studies. ${ }^{18}$ Such highly transparent, highly conducting ITO nanowires and vertical nanowire arrays will be ideally suited for devices that require transparent electrodes with large surface areas such as solar cells. Furthermore, transparent conducting films composed of ITO nanowires will be compatible with devices that cannot sustain hightemperature thin-film deposition processes (such as organic based devices), due to the ability to separate the hightemperature nanowire growth and low-temperature device fabrication processes. ${ }^{23}$

The observed resistivities of the ITO nanowires are several orders lower than those of $\operatorname{In}_{2} \mathrm{O}_{3}$ nanowires without intentional doping (on the order of $1 \Omega \cdot \mathrm{cm}$, data not shown) and indicate that $\mathrm{Sn}$ is indeed effectively incorporated in the ITO 

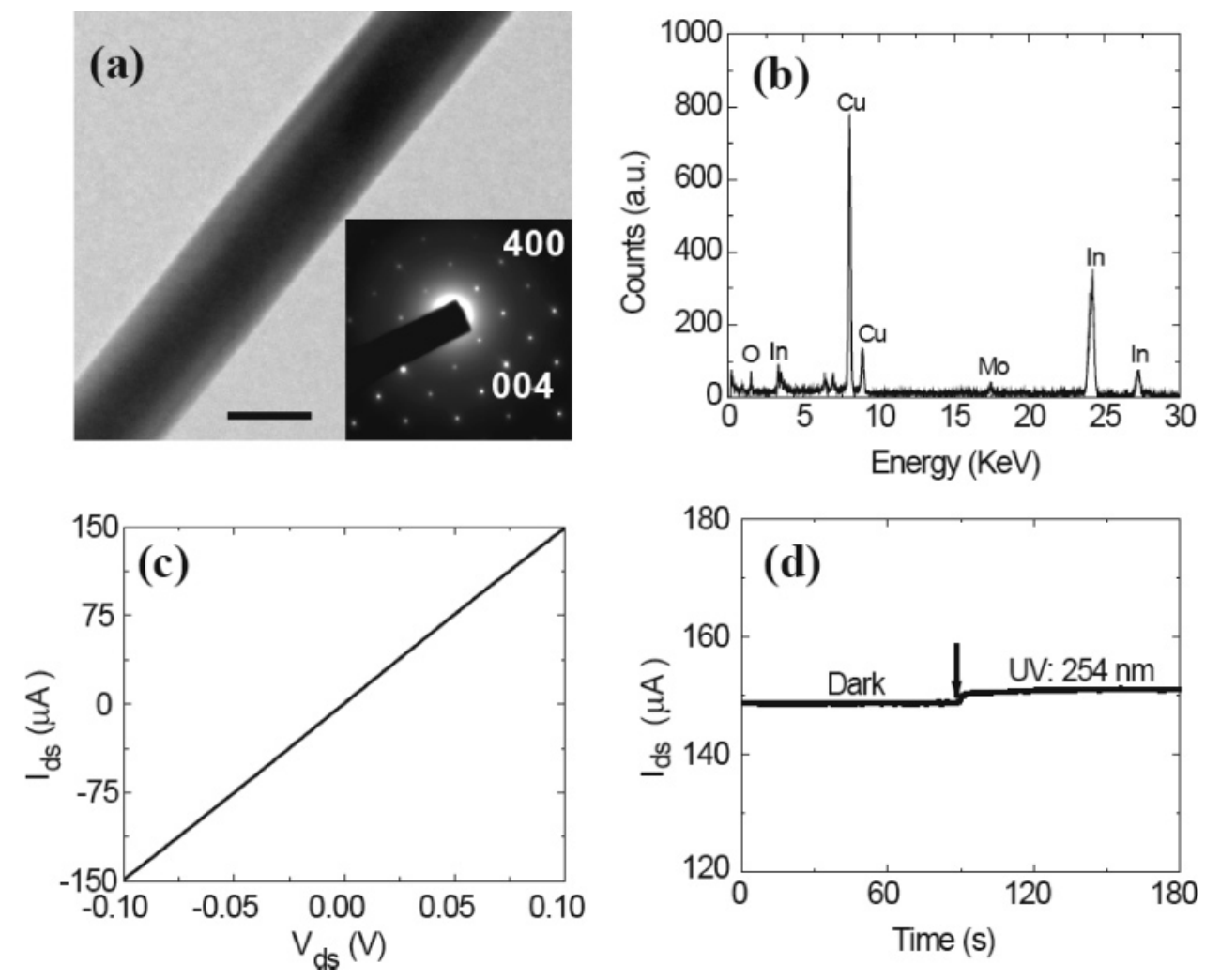

Figure 6. (a) TEM image of a single Mo-doped $\mathrm{In}_{2} \mathrm{O}_{3}$ (IMO) nanowire. Scale bar: $50 \mathrm{~nm}$. Inset, selected area diffraction pattern of the same nanowire. (b) Energy dispersive X-ray spectrum of an individual IMO nanowire. (c) $I_{\mathrm{ds}}-V_{\mathrm{ds}}$ curve of a typical IMO nanowire device with lateral size of $100 \mathrm{~nm}$ and length of $2.43 \mu \mathrm{m}$. (d) UV light response of the same IMO nanowire device.

nanowires, consistent with the EDS observation. Such high Sn doping levels will in fact render the ITO nanowires degenerately doped. Indeed, metal-like behaviors were observed in these ITO nanowire devices, as shown in Figure 5. Figure 5a shows the gate response curve of an ITO nanowire device with $90 \mathrm{~nm}$ lateral size and $3.2 \mu \mathrm{m}$ channel length. The current $I_{\mathrm{ds}}$ shows very little relative change as the gate voltage $V_{\mathrm{g}}$ is changed from -8 to $4 \mathrm{~V}$. As a firstorder estimation, the carrier density of the ITO nanowire can be calculated to be $\sim 4.3 \times 10^{20} \mathrm{~cm}^{-3}$ from the slope of the $I_{\mathrm{ds}}-V_{\mathrm{g}}$ curve. Such high carrier densities easily put the ITO nanowires in the degenerately doped regime. Assuming all $\mathrm{Sn}$ atoms are activated at room temperature, the $\mathrm{Sn}: \mathrm{In}$ ratio can then be estimated to be 1.4:100, on the same order of the EDS estimations. Considering the simplicity of the model being used to calculate the carrier density and the small Sn signal used in the EDS estimations, such level of agreement is remarkable and verifies that a Sn-doping level of a few percent has been achieved in the ITO nanowires. The high doping level also explains the lack of UV response of these ITO nanowire devices (Figure 5b) and the insensitivity to the ambient environment (see Supporting Information). Contrary to undoped $\operatorname{In}_{2} \mathrm{O}_{3}$ nanowires which are very sensitive to UV light due to photogeneration of carriers, ${ }^{24,25}$ the ITO nanowire device shows only $\sim 1.0 \%$ change in conductance when illuminated by UV light, even though the photon energy of the applied UV source $(254 \mathrm{~nm}, 4 \mathrm{~W})$ is clearly above the band gap of ITO. This lack of UV response is a direct result of the high starting carrier concentration. The metallic behavior was further verified by temperature- dependence measurements (Figure 5c), in which the resistivity of the ITO nanowire increases linearly from $2.66 \times 10^{-4}$ to $2.99 \times 10^{-4} \Omega \cdot \mathrm{cm}$ as the temperature is increased from 300 to $470 \mathrm{~K}$. This behavior agrees well with the expected linear resistivity-temperature relationship at high temperatures for a metal when scattering is dominated by electronacoustic phonon scattering. ${ }^{26}$ Detailed temperature dependence studies on another device with room-temperature resistivity of $6.69 \times 10^{-5} \Omega \cdot \mathrm{cm}$ show that the resistivity of the ITO nanowires over a wide temperature range (50-300 $\mathrm{K})$ can be well described by the Block-Grüneisen formula (see Supporting Information), as expected for a metal or a degenerately doped semiconductor. ${ }^{27}$ Finally, these "metallic" ITO nanowires can also carry a very high current density due to the single-crystalline nature. As shown in Figure 5d, the ITO nanowire device can endure a current of $2.5 \mathrm{~mA}$ before failure, corresponding to a current density of $3.1 \times$ $10^{7} \mathrm{~A} / \mathrm{cm}^{2}$. We note this failure current density is comparable to that obtained from metallic $\mathrm{NiSi}$ and $\mathrm{TaSi}_{2}$ nanowires. ${ }^{13,14}$ Inspections of the failed devices (Inset of Figure 5d) show failure occurred in the middle of the device, indicating that the failure of the ITO nanowires at high currents is caused by melting related to resistive self-heating instead of electromigration, a phenomenon also observed in metallic $\mathrm{NiSi}$ and TaSi nanowires. ${ }^{13,14}$

Besides ITO, Mo-doped indium oxide (IMO) films have been studied in the past as transparent conductors due to the high electron mobility. ${ }^{28} \mathrm{We}$ were able to obtain singlecrystalline IMO nanowires using the same VLS growth method, by replacing $\mathrm{SnO}$ with metal Mo during nanowire 
(a)

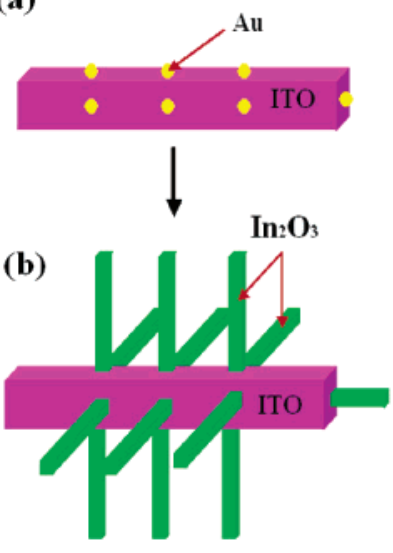

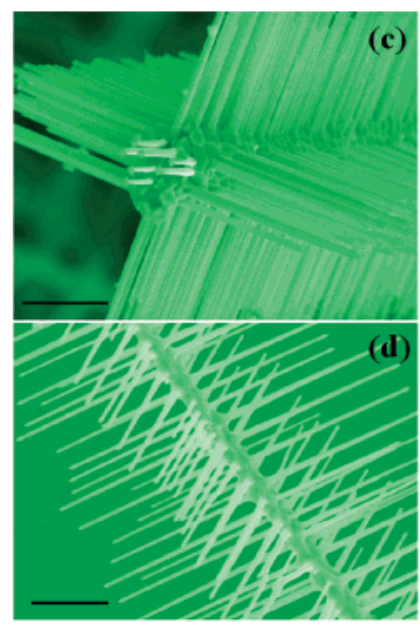

Figure 7. $(a, b)$ Schematics of the growth processes of branched $\mathrm{In}_{2} \mathrm{O}_{3}$ nanowires, showing the deposition of Au catalysts on ITO backbones (a) and the subsequent growth of $\operatorname{In}_{2} \mathrm{O}_{3}$ nanowire branches in a second VLS growth process (b). (c, d) SEM images of branched $\mathrm{In}_{2} \mathrm{O}_{3}$ nanowires grown on ITO nanowire backbones. The thickness of the Au catalyst is $10 \mathrm{~nm}$ in (c) and $2 \mathrm{~nm}$ in (d). Scale bars: $500 \mathrm{~nm}$.

growth. The IMO nanowires were grown at $1100{ }^{\circ} \mathrm{C}$ for 4 h. A representative TEM image of a single IMO nanowire with a cross-section size of $100 \mathrm{~nm}$ is shown in Figure 6a. The SAED pattern of the IMO nanowire (inset) reveals the single-crystal cubic structure and the [100] growth direction. The incorporation of Mo was verified by EDS analysis (Figure 6b). The electrical properties of the IMO nanowires were investigated and were found to be similar to those of the ITO nanowires: linear $I_{\mathrm{ds}}-V_{\mathrm{ds}}$ curves were observed in all devices studied (Figure 6c), and the IMO nanowire devices show very small response (about $1.0 \%$ ) to UV light (Figure 6d). The resistivity of the IMO nanowire in parts c and d of Figure 6 was estimated to be $2.74 \times 10^{-4} \Omega \cdot \mathrm{cm}$.

Finally, the ability to epitaxially grow nanowires on a suitable substrate enables us to fabricate branched semiconducting/metallic nanowire structures. Branched nanowires offer a possible route for three-dimensional integration of nanowire devices ${ }^{29}$ and have been reported on $\mathrm{Si}, \mathrm{GaP}$, and $\mathrm{ZnS}$ nanowire systems via a controlled multistep catalystmediated VLS process. ${ }^{30-32}$ Figure 7 a shows a schematic illustration of the two-step growth process used in our approach to obtain branched $\operatorname{In}_{2} \mathrm{O}_{3}$ nanowire arrays on ITO nanowires. Briefly, after the growth of ITO nanowires, $\mathrm{Au}$ catalyst nanoclusters were deposited via dc sputtering on the ITO nanowire "backbones", which then serve as substrates for the subsequent epitaxial growth of $\operatorname{In}_{2} \mathrm{O}_{3}$ nanowires in the second VLS growth process. Due to the excellent epitaxial relationship between $\mathrm{In}_{2} \mathrm{O}_{3}$ and ITO nanowires, three-dimensional (3D) growth of $\operatorname{In}_{2} \mathrm{O}_{3}$ nanowire arrays were readily obtained on the ITO backbones, as seen in parts c and $d$ of Figure 7. Furthermore, the density of the $\operatorname{In}_{2} \mathrm{O}_{3}$ nanowire branches can be controlled by adjusting the thickness of the deposited Au film. As shown in Figure 7, the density of the $\operatorname{In}_{2} \mathrm{O}_{3}$ nanowire branches is reduced dramatically when the Au film thickness is reduced from $10 \mathrm{~nm}$ (Figure 7c) to $2 \mathrm{~nm}$ (Figure 7d). This method for controlled growth of 3D hybrid metal/semiconductor nanowire structures may provide a rational route for the eventual large-scale integration of nanowire devices/sensors.

In conclusion, single-crystalline Sn-doped $\operatorname{In}_{2} \mathrm{O}_{3}$ and Modoped $\operatorname{In}_{2} \mathrm{O}_{3}$ nanowires were grown by a vapor transport method. Optical and electrical investigations demonstrated that these nanowires are transparent conductors with resistivities as low as $6.29 \times 10^{-5} \Omega \cdot \mathrm{cm}$ and failure-current densities as high as $3.1 \times 10^{7} \mathrm{~A} / \mathrm{cm}^{2}$. Hierarchically branched semiconducting $\mathrm{In}_{2} \mathrm{O}_{3}$ nanowire arrays with different densities were also self-assembled on these transparent conducting nanowire backbones in a second VLS growth process and demonstrated a rational route for nanowire devices integration in three dimensions.

Acknowledgment. We thank J. Guo and J. Phillips for helpful discussions. This work was supported in part by the National Science Foundation (ECS-0601478). This work used the Michigan Nanofabrication Facility (MNF) at UM, a member of the National Nanotechnology Infrastructure Network (NNIN) funded by the NSF.

Supporting Information Available: X-ray diffraction spectrum of the ITO buffer layer, $I_{\mathrm{ds}}-V_{\mathrm{ds}}$ curves of the ITO nanowire devices in ambient air vs in vacuum and detailed temperature-dependent studies of a four-probe ITO nanowire device. This material is available free of charge via the Internet at http://pubs.acs.org.

\section{References}

(1) Lieber, C. M. MRS Bull. 2003, 28, 486.

(2) Xiang, J.; Lu, W.; Hu, Y. J.; Wu, Y.; Yan, H.; Lieber, C. M. Nature 2006, 441, 489.

(3) Law, M.; Goldberger, J.; Yang, P. D. Annu. Rev. Mater. Res. 2004, $34,83$.

(4) Xia, Y. N.; Yang, P. D.; Sun, Y. G.; Wu, Y. Y.; Mayers, B.; Gates, B.; Yin, Y. D.; Kim, F.; Yan, Y. Q. Adv. Mater. 2003, 15, 353.

(5) Fan, H. J.; Werner, P.; Zacharias, M. Small 2006, 2, 700.

(6) Lu, J. G.; Chang, P. C.; Fan, Z. Y. Mater. Sci. Eng. R 2006, 52, 49.

(7) Wang, Z. L. Annu. Rev. Phys. Chem. 2004, 55, 159.

(8) Li, C.; Zhang, D. H.; Han, S.; Liu, X. L.; Tang, T.; Zhou, C. W. Adv. Mater. 2003, 15, 143.

(9) Minami, T. Semicond. Sci. Technol. 2005, 20, S35.

(10) Park, S. K.; Han, J. I.; Kim, W. K.; Kwak, M. G. Thin Solid Films 2001, 397, 49.

(11) Rozati, S. M.; Ganj, T. Renewable Energy 2004, 29, 1671.

(12) Lewis, J.; Grego, S.; Chalamala, B.; Vick, E.; Temple, D. Appl. Phys. Lett. 2004, 85, 3450 .

(13) Wu, Y.; Xiang, J.; Yang, C.; Lu, W.; Lieber, C. M. Nature 2004, $430,61$.

(14) Chueh, Y. L.; Ko, M. T.; Chou, L. J.; Chen, L. J.; Wu, C. S.; Chen, C. D. Nano Lett. 2006, 6, 1637.

(15) Pan, Z. W.; Dai, Z. R; Wang, Z. L. Science 2001, 291, 1947.

(16) Li, S. Y.; Lee, C. Y.; Lin, P.; Tseng, T. Y. Nanotechnology 2005, $16,451$.

(17) Kalyanikutty, K. P.; Gundiah, G.; Edem, C.; Govindaraj, A.; Rao, C. N. R. Chem. Phys. Lett. 2005, 408, 389.

(18) Wan, Q.; Song, Z. T.; Feng, S. L.; Wang, T. H. Appl. Phys. Lett. 2004, 85, 4759.

(19) Peng, X. S.; Meng, G. W.; Wang, X. F.; Wang, Y. W.; Zhang, J.; Liu, X.; Zhang, L. D. Chem. Mater. 2002, 14, 4490.

(20) Orlandi, M. O.; Aguiar, R.; Lanfredi, A. J. C.; Longo, E.; Varela, J. A.; Leite, E. R. Appl. Phys. A 2005, 80, 23.

(21) Wan, Q.; Wei, M.; Zhi, D.; MacManus-Driscoll, J. L.; Blamire, M. G. Adv. Mater. 2006, 18, 234.

(22) Kim, H.; Horwitz, J. S.; Kim, W. H.; Kafafi, Z. H.; Chrisey, D. B. J. Appl. Phys. 2002, 91, 5371.

(23) McAlpine, M. C.; Friedman, R. S.; Jin, S.; Lin, K.; Wang, W. U.; Lieber, C. M. Nano Lett. 2003, 3, 1531 . 
(24) Zhang, D. H.; Liu, Z. Q.; Li, C.; Tang, T.; Liu, X. L.; Han, S.; Lei, B.; Zhou, C. W. Nano Lett. 2004, 4, 1919.

(25) Zhang, D. H.; Li, C.; Han, S.; Liu, X. L.; Tang, T.; Jin, W.; Zhou, C. W. Appl. Phys. A 2003, 77, 163.

(26) Ashcroft, N. W.; Mermin, N. D. Solid State Physics; Brooks Cole: New York, 1976.

(27) Ziman, J. M. Electrons and Phonons; Clarendon Press: Oxford, 1960

(28) Warmsingh, C.; Yoshida, Y.; Readey, D. W.; Teplin, C. W.; Perkins, J. D.; Parilla, P. A.; Gedvilas, L. M.; Keyes, B. M.; Ginley, D. S. J. Appl. Phys. 2004, 95, 3831.
(29) Wang, D. L.; Lieber, C. M. Nat. Mater. 2003, 2, 355.

(30) Wang, D. L.; Qian, F.; Yang, C.; Zhong, Z. H.; Lieber, C. M. Nano Lett. 2004, 4, 871.

(31) Dick, K.; Deppert, K.; Larsson, M.; Martensson, T.; Seiffert, W.; Wallenberg, L. R.; Samuelson, L. Nat. Mater. 2004, 3, 380.

(32) Jiang, Y.; Zhang, W. J.; Jie, J. S.; Meng, X. M.; Zapien, J. A.; Lee, S. T. Adv. Mater. 2006, 18, 1527

NL062213D 